\title{
Tamoxifen with ovarian function suppression versus tamoxifen alone as an adjuvant treatment for premenopausal breast cancer: a meta-analysis of published randomized controlled trials
}

\author{
This article was published in the following Dove Press journal: \\ OncoTargets and Therapy \\ 12 June 2015 \\ Number of times this article has been viewed
}

\author{
Shunchao Yan' \\ Kai Li' \\ Xin Jiao ${ }^{2}$ \\ Huawei Zou' \\ 'Department of Oncology, Shengjing \\ Hospital of China Medical University, \\ ${ }^{2}$ Department of Respiratory Medicine, \\ Shenyang Chest Hospital, Shenyang, \\ People's Republic of China
}

\begin{abstract}
Background: Ovarian function suppression(OFS) significantly downregulates the concentration of plasma estrogens. However, it is unclear whether it offers any survival benefits if combined with adjuvant tamoxifen treatment in premenopausal women. This meta-analysis was designed to assess data from previous studies involving adjuvant tamoxifen treatment plus OFS in premenopausal breast cancer.
\end{abstract}

Methods: Electronic literature databases (PubMed, Embase, the Web of Science, and the Cochrane Library) were searched for relevant randomized controlled trials published prior to February 1, 2015. Only randomized controlled trials that compared tamoxifen alone with tamoxifen plus OFS for premenopausal women with breast cancer were selected. The evaluated endpoints were disease-free survival and overall survival.

Results: Four randomized controlled trials comprising 6,279 patients (OFS combination, $\mathrm{n}=3,133$; tamoxifen alone, $\mathrm{n}=3,146$ ) were included in the meta-analysis. There was no significant improvement in disease-free survival or overall survival with addition of OFS in either the whole population or the hormone receptor-positive subgroup. The risk of distant recurrence was not reduced with the addition of OFS in the whole population. A subgroup analysis showed that addition of OFS significantly improved overall survival in patients who were administered chemotherapy.

Conclusion: Based on the available studies, concurrent administration of OFS and adjuvant tamoxifen treatment for premenopausal women with breast cancer has no effect on prolonging disease-free survival and overall survival, excluding patients who were administered chemotherapy. It should not be widely recommended, except perhaps for women who were hormonereceptor positive and who were also administered adjuvant chemotherapy.

Keywords: tamoxifen, ovarian function suppression, breast cancer, premenopausal women, adjuvant treatment, meta-analysis

\section{Introduction}

Breast cancer is still the leading cause of cancer-related death for women aged 20-59 years. ${ }^{1}$ Approximately $25 \%$ of breast cancer patients are younger than 50 years of age. ${ }^{2}$ Sixty percent of premenopausal patients with breast cancer are estrogen receptor (ER)-positive. ${ }^{3}$ Estrogen can enhance the proliferation of ER-positive breast cancer cells. Estrogens are mainly secreted by the ovaries in premenopausal women. Tamoxifen, a selective ER modulator, acts as an antagonist of estrogen in breast cancer. Tamoxifen is now the standard adjuvant endocrine therapy for ER-positive breast cancer in premenopausal women. Since 1990, mortality related to breast cancer has

\footnotetext{
Correspondence: Shunchao Yan; Huawei Zou

Department of Oncology, Shengjing Hospital of China Medical University, Shenyang I I0022, People's Republic of China

Tel +86249661563115

Fax +86 249661563115

Email yanshunchao666@163.com; Zouhw0@I26.com
}

submit your manuscript | www.dovepress.com

Dovepress

http://dx.doi.org/10.2147/OTT.S86817 
decreased by over $25 \%$, partly because of tamoxifen-based endocrine therapies. ${ }^{4}$

It has been reported that tamoxifen could increase plasma estradiol levels three to four times over pretreatment levels because of the stimulatory effects of tamoxifen on pituitaryovarian function. ${ }^{5,6}$ High plasma estradiol concentrations might partially weaken the effect of tamoxifen by competing for binding sites on ERs. Suppression of ovarian estrogen synthesis is a potential endocrine strategy. It is believed that ovarian function suppression (OFS) in combination with tamoxifen in premenopausal women is a more efficacious hormonal therapy for ER-positive breast cancer than tamoxifen alone. In advanced breast cancer, addition of OFS to tamoxifen prolongs progression-free survival and overall survival (OS) ${ }^{6}$ The question of whether there are any advantages to combining OFS with tamoxifen as an adjuvant treatment for premenopausal women has garnered a great deal of interest but has not yet been answered. Several studies have attempted to answer this question, ${ }^{7-9}$ but the results have been inconsistent. Sequential meta-analyses have reviewed adjuvant endocrine strategies for breast cancer, but none have focused on addition of ovarian suppression to adjuvant tamoxifen treatment for premenopausal women. ${ }^{10-13}$

Recently, the E-3193 and SOFT (Suppression of Ovarian Function Trial) trials have provided additional data. ${ }^{14,15}$ However, these studies were limited because of their sample size and were not definitive enough to generate guidelines for oncologists. As such, we believe a meta-analysis is necessary to help guide therapeutic decisions. To address these issues, we performed a meta-analysis to compare OFS plus tamoxifen with tamoxifen alone as an adjuvant treatment for premenopausal women.

\section{Materials and methods}

\section{Search strategy and selection of studies}

We searched medical electronic databases, including PubMed, Embase, the Web of Science, and the Cochrane Register of Controlled Trials (CENTRAL) using keywords including "breast cancer", "tamoxifen”, "ovarian function suppression", "luteinizing hormone-releasing hormone agonist", "LHRH", and "randomized" to identify all randomized controlled trials that compared adjuvant tamoxifen treatment with tamoxifen plus OFS in premenopausal patients with breast cancer (last search updated on February 1, 2015). Eligible methods to achieve OFS included oophorectomy, ovarian irradiation, or luteinizing hormone-releasing hormone agonists. If a trial had more than two treatment arms, a valid pairwise comparison was considered separately.
Studies that used other concomitant anticancer treatments were eligible if these treatments did not differ systematically between the investigated arms. First, the titles and abstracts of the articles were read to exclude irrelevant studies. Inclusion of trials was then decided based on the full text of the remaining articles. In addition, the reference lists of all retrieved articles were manually searched to identify other potentially relevant trials.

\section{Data extraction}

Two authors (SY and XJ) performed the study selection, data extraction, and data entry independently. Inclusion of a study was done by consensus. The following information was recorded for each study: author; name of study; recruitment period; entry criteria (stage); hormone receptor (HR) status; cohort size and study design; treatment information (including treatment regimens in control and experimental arms); and outcomes (rates or number of events) for OS, disease-free survival (DFS), and distant recurrence. DFS was defined as time from randomization to first appearance of one of the following: invasive recurrence of breast cancer (local, regional, or distant), invasive contralateral breast cancer, second (non-breast) invasive cancer, or death without breast cancer recurrence or second invasive cancer. OS was defined as time from randomization to death from any cause. Distant recurrence was defined as a cancer recurrence at distant sites, excluding the ipsilateral chest wall and breast. The studies were assessed for quality in accordance with the guidelines in the Cochrane reviewers' handbook. ${ }^{16}$

\section{Statistical analysis}

The data analyses were performed using RevMan version 5.2 (free software downloaded from http://www. cochrane.org). All analyses were carried out on an intentionto-treat basis. The number of events per arm was used to calculate risk ratios (RRs) and their 95\% confidence intervals (CIs), which are presented in a Forest plot. A chi-squarebased $Q$ statistic was used to test for heterogeneity. A $P$-value of $<0.10$ was considered to be statistically significant. The $I^{2}$ statistic was used to examine the extent of heterogeneity (considered large for $I^{2}$ values of 50\%-74\% and very large for $I^{2}$ values of $\left.\geq 75 \%\right) .{ }^{17} \mathrm{~A}$ random-effects model was used if the test for heterogeneity was significant; otherwise, the fixed-effects model was used. Publication bias was evaluated using Begg's and Egger's tests. Probable significant publication bias was considered with a $P$-value of $<0.05$. The Begg's and Egger's tests were performed using Stata version 12.0 (Stata Corp LP, College Station, TX, USA). 


\section{Results}

\section{Description of studies}

As shown in Figure 1, we identified 486 potentially relevant articles in the primary literature search. After removing duplicate entries, 452 articles remained. After screening the titles and/or abstracts, 476 studies were excluded because they did not meet the inclusion criteria for this study, including laboratory studies and case reports. Of the ten remaining articles, four did not include tamoxifen alone arms, ${ }^{18-21}$ while two did not provide adequate survival data. ${ }^{7,22}$ As a result, four randomized Phase III trials were eligible for this meta-analysis. ${ }^{8,9,14,15}$ Table 1 summarizes the designs and characteristics of the included studies. The ZIPP (Zoladex in Premenopausal Patients) trial randomly assigned 2,710 women who were premenopausal or aged younger than 50 years with operable stage I or II breast cancer to goserelin for 2 years, tamoxifen for 2 years, combined treatment, or no further treatment in a $2 \times 2$ factorial design. ${ }^{8} \mathrm{We}$ concentrated on the results associated with the effect of combining goserelin with tamoxifen, so we extracted data for the tamoxifen alone and goserelin plus tamoxifen arms. The Adjuvant Breast Cancer (ABC) Ovarian Ablation or Suppression $[\mathrm{ABC}(\mathrm{OAS})]$ trial randomly assigned premenopausal patients with early-stage (T1-3a N0-1 M0) breast cancer to a tamoxifen alone group or a tamoxifen plus OFS group. ${ }^{9}$ The Eastern Cooperative Oncology Group conducted a Phase III trial (E-3193) comparing tamoxifen alone with tamoxifen plus OFS in premenopausal women. The enrolled patients had node-negative, HR-positive breast cancer, and adjuvant chemotherapy was not permitted. The trial was terminated before reaching the enrollment goal because of slow accrual. ${ }^{14}$ SOFT is a Phase III trial that randomly assigned patients to tamoxifen alone, tamoxifen with OFS, or exemestane (an aromatase inhibitor) with OFS. ${ }^{15}$ Data for the tamoxifen alone and tamoxifen plus OFS arms were extracted for this analysis. The median patient age in the four included studies was 44, 43, 45, and 43 years (Table 1). The median follow-up duration for all trials was $>5$ years. The number of patients ranged from 337 to 2,144 , and the total number of patients analyzed was 6,279.

\section{Effect of additional OFS with tamoxifen on DFS and OS of the whole population}

The four included studies all provided DFS data. The analysis showed no significant between-study heterogeneity ( $\left.P=0.82 ; I^{2}=0 \%\right)$, and a fixed-effects model was used. The meta-analysis results showed that administration of OFS was not significantly associated with improved DFS (versus tamoxifen alone: $\mathrm{RR}=0.92 ; 95 \% \mathrm{CI} 0.84-1.00 ; P=0.06$; Figure 2A). These four studies provided OS data. A fixedeffects model was used because of a lack of heterogeneity between the studies $\left(P=0.85 ; I^{2}=0 \%\right)$. The combined RR regarding OS for tamoxifen plus OFS versus tamoxifen alone was 0.91 (95\% CI $0.80-1.03 ; P=0.15$, Figure 2B). The results showed that addition of OFS to tamoxifen did not improve OS.

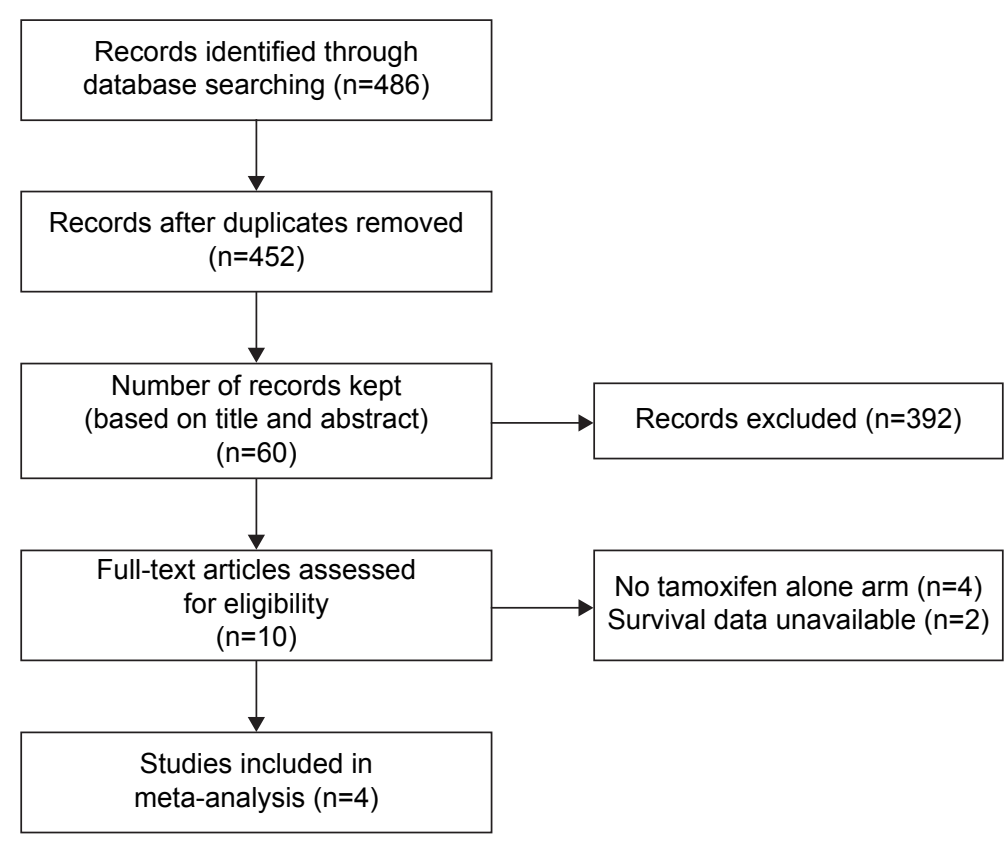

Figure I Flow diagram of study selection. 


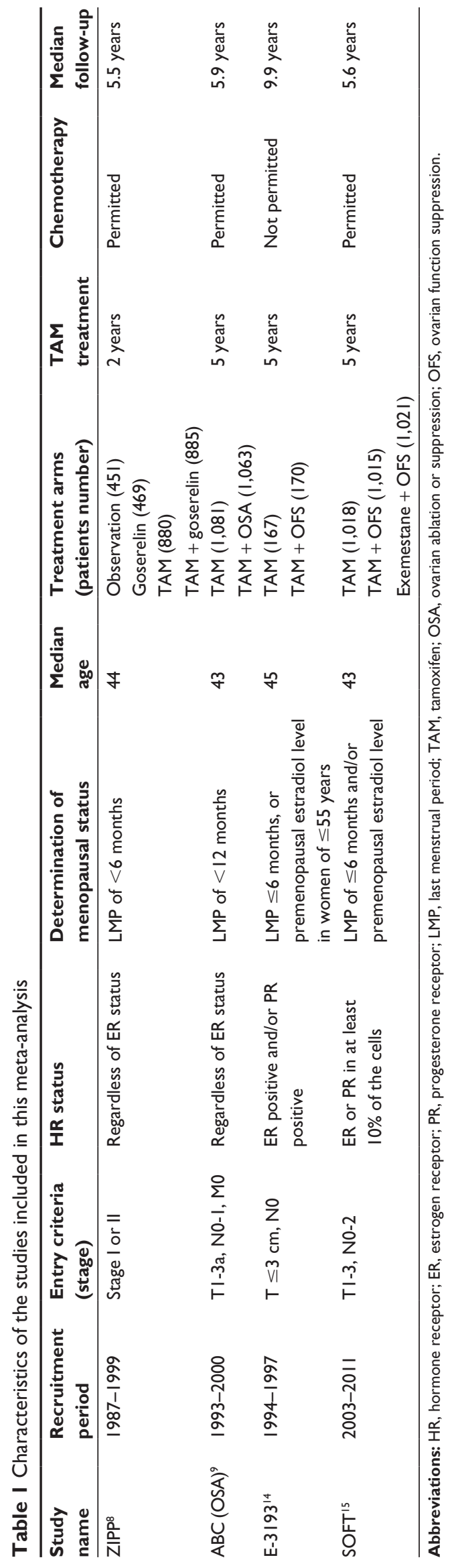

\section{Effect of additional OFS on outcomes in patient subgroups}

We performed a subgroup meta-analysis based on HR status and chemotherapy experience. Patients were enrolled regardless of HR status, and their ER status was detected after enrollment in the ZIPP and ABC (OAS) trials. Both SOFT and the E-3193 trial enrolled only patients who were ER-positive, progesterone receptor-positive, or both, and provided DFS data. A fixed-effects model was used because no heterogeneity existed between the studies $(P=0.96$; $\left.I^{2}=0 \%\right)$. The meta-analysis results showed that the combined RR for tamoxifen plus OFS versus tamoxifen alone was 0.87 (0.71-1.06; $P=0.16$; Figure $3 \mathrm{~A})$. The ZIPP trial provided OS data for the ER-positive subgroup. The SOFT and E-3193 trials provided OS data for patients who were ER-positive, progesterone receptor-positive, or both. We extracted OS data for the ER subgroup from the ZIPP trial and OS data from the SOFT and E-3193 trials. No significant between-study heterogeneity was found ( $P=0.93 ; I^{2}=0 \%$ ), so the fixed-effects model was used. The meta-analysis results showed that the combined RR for OS regarding tamoxifen plus OFS versus tamoxifen alone was 0.84 (95\% CI $0.66-1.07$; $P=0.16$; Figure $3 \mathrm{~B}$ ). The results showed that addition of OFS to tamoxifen did not improve DFS or OS in the HR-positive subgroup.

Adjuvant chemotherapy was not permitted in the E-3193 study. Both the SOFT and ZIPP trials provided OS data for the chemotherapy and non-chemotherapy subgroups. The fixed-effects model was used for patients who were not administered chemotherapy because there was no significant between-study heterogeneity $\left(P=0.20 ; I^{2}=39 \%\right)$. The meta-analysis results showed that the combined RR for tamoxifen plus OFS versus tamoxifen alone was 1.10 (95\% CI $0.80-1.51 ; P=0.55$; Figure 4A). The fixed-effects model was also used for patients who were administered chemotherapy because no significant heterogeneity was found $\left(P=0.48 ; I^{2}=0 \%\right)$. The combined RR for tamoxifen plus OFS versus tamoxifen alone was 0.76 (95\% CI 0.60-0.97; $P=0.03$; Figure 4B). The results showed that addition of OFS to tamoxifen significantly improved OS in the chemotherapy subgroup, but not in the non-chemotherapy subgroups.

Both the $\mathrm{ABC}(\mathrm{OAS})$ and SOFT studies provided distant recurrence data for the tamoxifen and combined OFS arms. The fixed-effects model was used because of no significant heterogeneity $\left(P=0.70 ; I^{2}=0 \%\right.$ ). The combined RR for tamoxifen plus OFS versus tamoxifen alone was 0.97 (95\% CI $0.86-1.10 ; P=0.68$; Figure $4 \mathrm{C})$. The results showed that addition of OFS to tamoxifen did not reduce distant recurrence. 


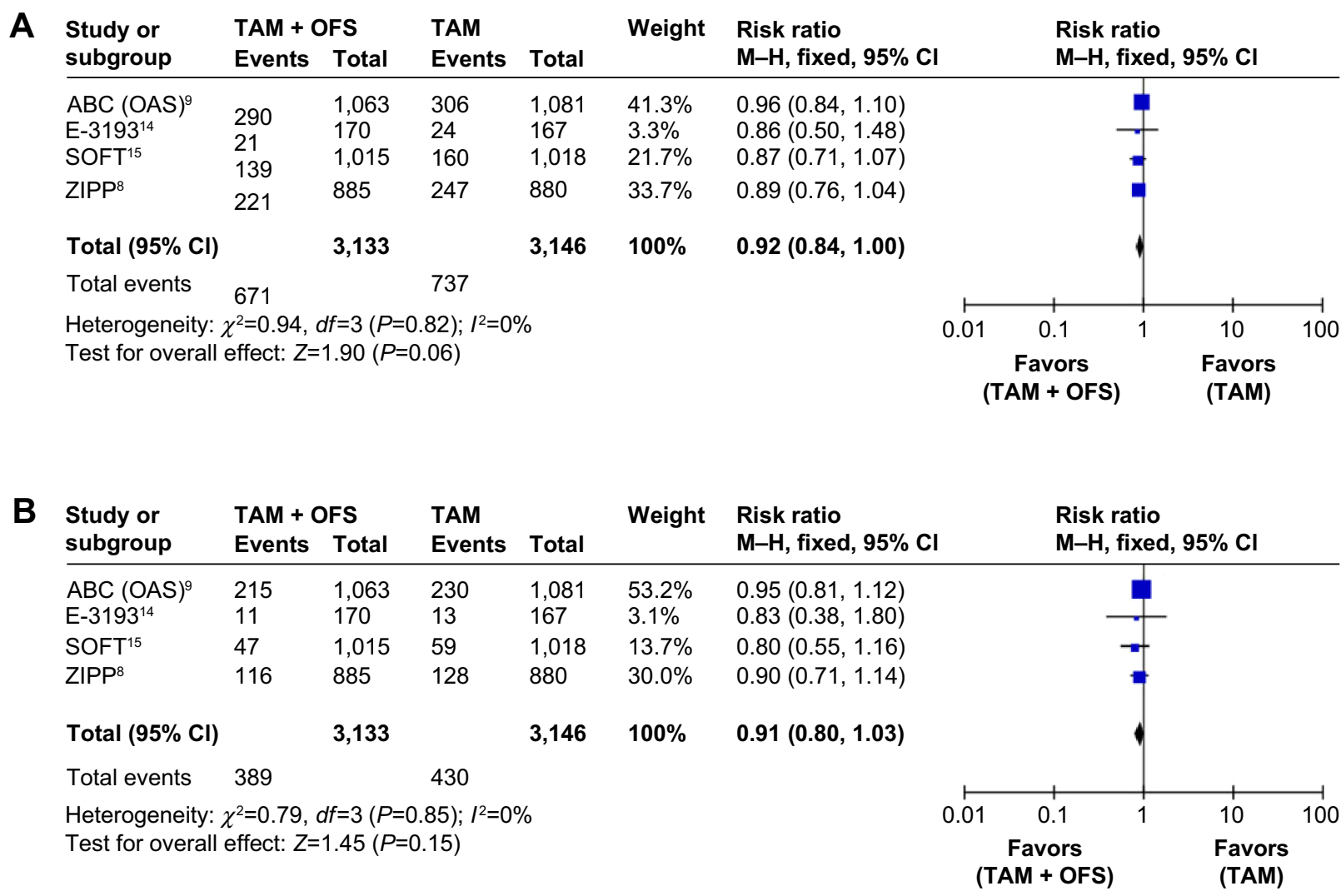

Figure 2 Forest plot of risk ratio for disease-free survival (A) or overall survival (B) in the whole population. Abbreviations: $\mathrm{Cl}$, confidence interval; TAM, tamoxifen; OFS, ovarian function suppression; $\mathrm{M}-\mathrm{H}$, Mantel-Haenszel.

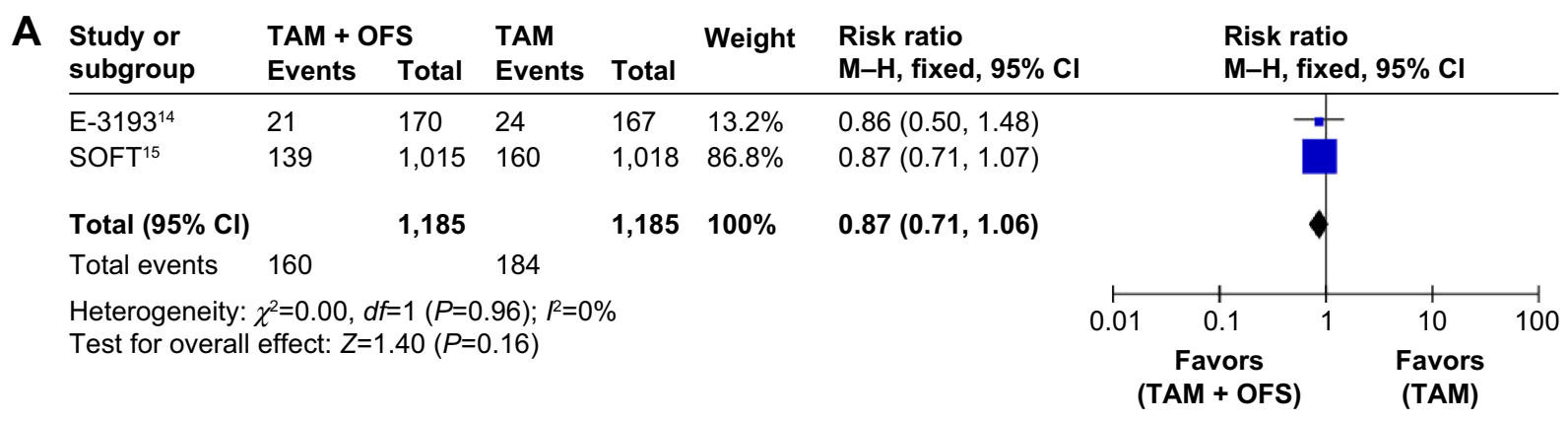

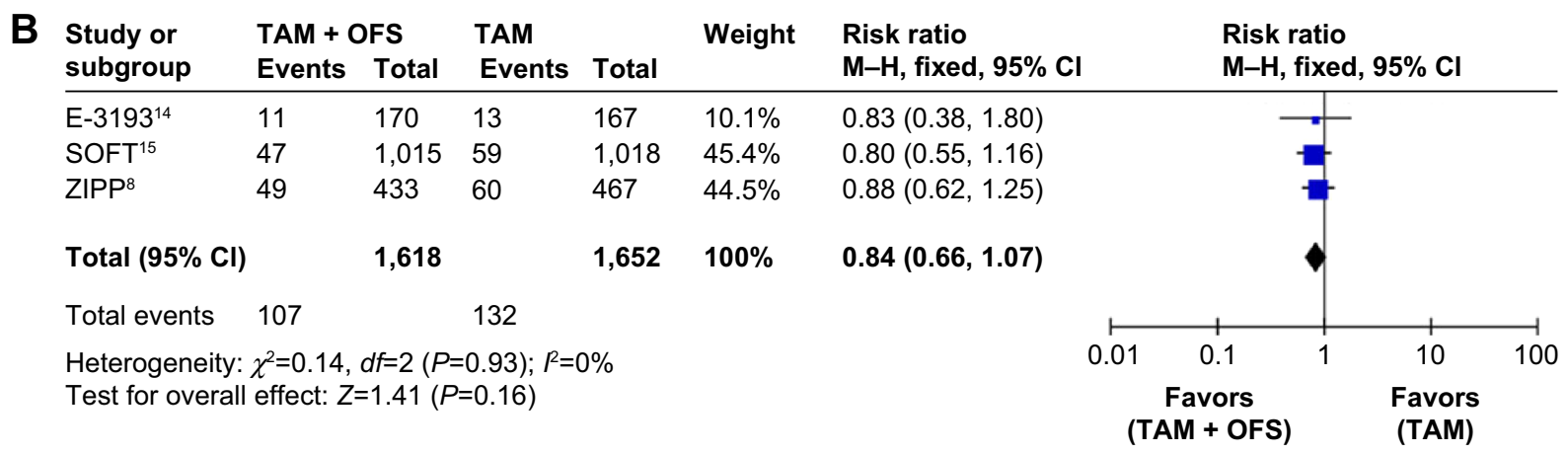

Figure 3 Forest plot of risk ratio for disease-free survival (A) and overall survival (B) among hormone receptor-positive patients.

Abbreviations: $\mathrm{Cl}$, confidence interval; TAM, tamoxifen; OFS, ovarian function suppression; $\mathrm{M}-\mathrm{H}$, Mantel-Haenszel. 


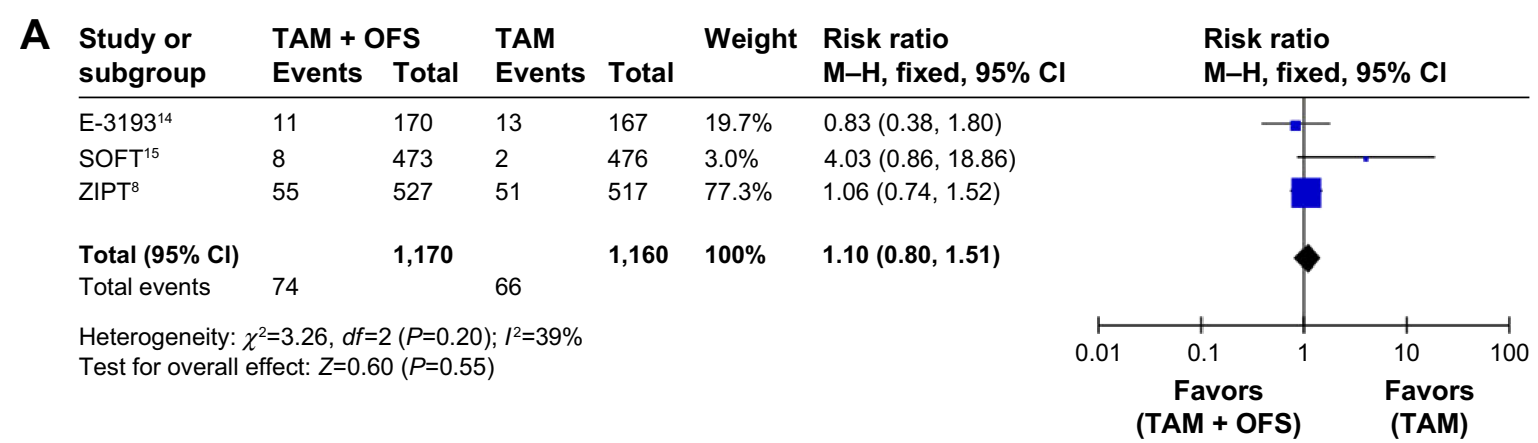

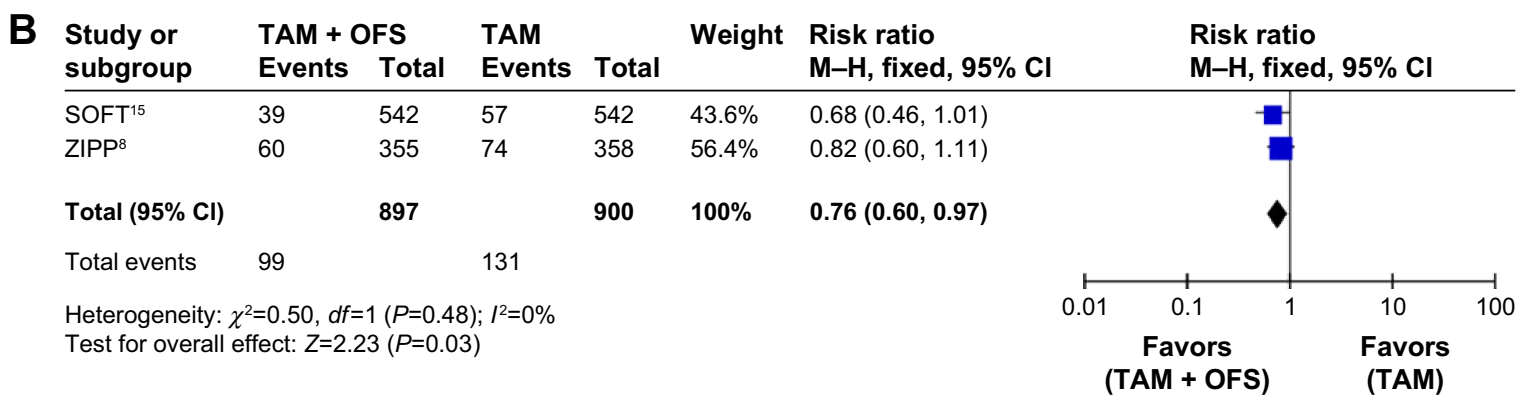

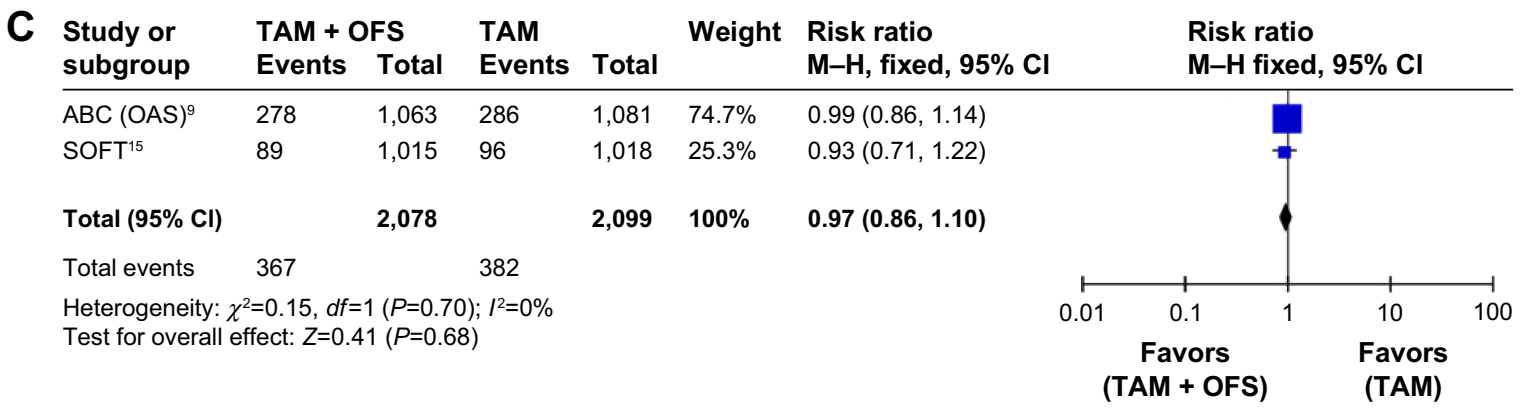

Figure 4 Forest plot of risk ratio for overall survival among patients who were administered no chemotherapy (A) or chemotherapy (B). Further, a forest plot of risk ratio for distant recurrence in the whole population (C).

Abbreviations: $\mathrm{Cl}$, confidence interval; TAM, tamoxifen; OFS, ovarian function suppression; M-H, Mantel-Haenszel.

\section{Publication bias}

There was no evidence of publication bias, as shown by inspection of a funnel plot for DFS (Figure 5A) and OS (Figure 5B). Four studies evaluating DFS yielded Begg and Egger $P$-values of 0.09 and 0.20 , respectively. Four studies evaluating OS yielded Begg and Egger $P$-values of 0.31 and 0.41 , respectively. Moreover, publication bias was not detected in the subgroup meta-analyses.
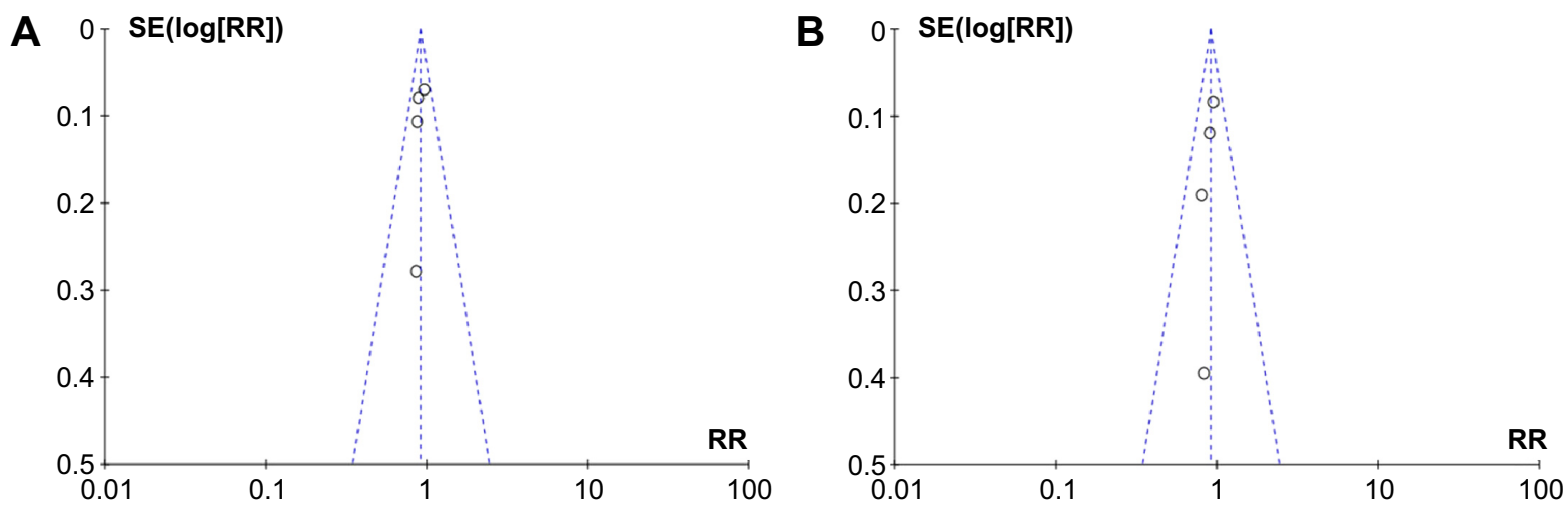

Figure 5 Funnel plot/scatter plots for disease-free survival $(\mathbf{A})$ and overall survival (B). The symmetric inverted funnel shape suggests that publication bias is unlikely. Abbreviations: SE, standard error; RR, risk ratio. 


\section{Discussion}

Tamoxifen was first reported by Cole et al for treating breast cancer in $1971,{ }^{23}$ and approved in the UK in 1973 and in the USA in $1977 .{ }^{24}$ At the beginning, it was used as a treatment for late recurrent or metastatic breast cancer. Tamoxifen has since been used also as an adjuvant treatment and preventive measure for HR-positive breast cancer. Several studies have shown that 5 years of adjuvant tamoxifen treatment is associated with a reduction in breast cancer recurrence and death, ${ }^{25}$ even at 15 years after diagnosis. ${ }^{26} \mathrm{~A}$ prolonged 10-year tamoxifen treatment appears to further reduce the risk of recurrence and mortality, as compared with a 5 -year tamoxifen treatment. ${ }^{27,28}$ The ovaries are the primary source of estrogens, which may enhance the proliferation of HR-positive breast cancer. Oophorectomy was first recommended for treatment of breast cancer by Albert Schinzinger in $1889 .{ }^{29}$ Compared with no adjuvant therapy, OFS reduces the risk of recurrence by $31 \%$ and mortality by $28 \% .{ }^{30}$ OFS and chemotherapy have demonstrated similar efficacy in premenopausal patients with early breast cancer if used alone. ${ }^{31}$ However, OFS did not significantly improve outcomes if added to adjuvant chemotherapy. ${ }^{32,33}$ For several years, it has been unclear to oncologists whether OFS provides any benefit beyond that offered by adjuvant tamoxifen treatment in premenopausal breast cancer. In the present study, we analyzed four studies published between 2006 and 2014, that comprised a total of 6,279 cases. The meta-analysis results showed an $8 \%$ reduction in recurrence after adding OFS, as compared with tamoxifen treatment alone, although this was not significant (RR 0.92; 95\% CI $0.84-1.00 ; P=0.06)$. There was also a $9 \%$ reduction in death, but this also did not reach statistical significance (RR 0.91; 95\% CI 0.80-1.03; $P=0.15$ ). The ZIPP trial and ABC (OAS) trial began enrolling patients in 1987 and 1993 , respectively, when HR testing was not routinely performed. Consequently, both the ZIPP and ABC (OAS) trials enrolled patients regardless of HR status. ${ }^{89}$ We conducted another subgroup meta-analysis for the HR-positive patients, but no significant improvement was demonstrated in DFS or OS from addition of OFS. The results suggest that adding OFS should not be commonly recommended as an adjuvant treatment for premenopausal women because it offers no additional benefits.

It has been reported that patients with HR-positive breast cancer might benefit from chemotherapy-induced downregulation of estrogen, because of chemotherapy-induced ovarian suppression. ${ }^{34}$ Further, the non-chemotherapy subgroup might obtain additional benefits from combining
OFS with tamoxifen. However, the present meta-analysis results show that addition of OFS significantly improved OS in the chemotherapy subgroup (a mortality reduction of $24 \% ; P=0.03$ ). However, it did not provide any benefits in the non-chemotherapy subgroup. The reason might be that patients who were administered adjuvant chemotherapy were in a subgroup considered to be at a relatively higher risk for adverse outcomes. ${ }^{35}$ Adjuvant endocrine therapy with tamoxifen alone could not sufficiently reduce the risk of recurrence and death, and the addition of OFS might be helpful. However, in some of these studies, the chemotherapy regimens included a combination of cyclophosphamide, methotrexate, and fluorouracil or its equivalent, which would be considered insufficient today. This was also a limitation of the present study. However, patients who were administered chemotherapy have similar characteristics and a higher risk of recurrence. Distant recurrence results mainly from systemic treatment failure. ${ }^{36,37}$ Both the $\mathrm{ABC}$ (OSA) and SOFT trials provided a distant recurrence rate for the tamoxifen alone arm and the tamoxifen plus OFS arm., ${ }^{915}$ The meta-analysis results showed that addition of OFS did not reduce distant recurrence. Additionally, age is a factor that may influence the results of tamoxifen plus OFS therapy. The ABC (OSA) trial showed that only patients under the age of 40 years might benefit from addition of OFS. However, the authors did not provide the original survival data of the age subgroups. ${ }^{9}$ The ZIPP trial showed that the effect of tamoxifen alone and tamoxifen plus OFS was similar in women aged 40 years and over. The 5 -year survival rate was $76.2 \%$ for patients assigned to tamoxifen alone and $82.6 \%$ for those assigned to tamoxifen plus OFS in women younger than 40 years, although the results were less conclusive because of the smaller number of events (one was contained within the $95 \%$ CIs which were somewhat wide). ${ }^{8}$ The SOFT trial showed that the freedom from breast cancer rate at 5 years was $67.7 \%$ for patients assigned to tamoxifen alone and $78.9 \%$ for those assigned to tamoxifen plus OFS in the very young subgroup ( $<35$ years).${ }^{15}$ The above results demonstrate that the young subgroup ( $<40$ years) might benefit from addition of OFS. However, the included studies did not contain an adequate number of subgroups based on age. Further, the age groups were divided based on different cut-off points (40 years or 35 years) and did not provide adequate and specific survival data. We could not conduct a subgroup meta-analysis based on age. This topic may need further studies in future.

The present study includes the latest published trials, and subgroup analyses were performed to ensure that the results are reliable and valid. However, our meta-analysis had some 
limitations. First, the results of the subgroup analyses were calculated based on two to three studies with a relatively small sample size. Second, the concomitant anticancer treatments used in the four trials were not exactly the same because of advances in chemotherapy. Third, there were differences in the criteria used for determination of menopausal status among the four trials. In addition, the SOFT trial included more patients than any of the rest of the studies, and had a relatively higher impact on the statistical results, which may reduce the reliability of this study.

OFS was carried out to help premenopausal women to achieve an established postmenopausal state. The ATAC (Arimidex, Tamoxifen, Alone or in Combination) and BIG (Breast International Group) 1-98 trials found that adjuvant therapy with an aromatase inhibitor was a better choice than tamoxifen in HR-positive postmenopausal breast cancer. ${ }^{38,39}$ The TEAM (Tamoxifen and Exemestane Adjuvant Multinational) trial found that exemestane monotherapy and sequential treatment (tamoxifen followed by exemestane) are two appropriate options for postmenopausal women with HR-positive early breast cancer. ${ }^{40}$ Further, analysis of data from TEXT (Tamoxifen and Exemestane Trial) and SOFT demonstrated that adjuvant exemestane plus OFS provided more benefits than adjuvant tamoxifen plus OFS, ${ }^{41}$ although the final results of TEXT have not been published. However, exemestane is more expensive than tamoxifen. Efficacy and cost should both be considered while planning treatment strategies. High costs might reduce patient compliance, which might lead to failure of endocrine therapy. Therefore, in low-income and middle-income countries, tamoxifen plus OFS might be an appropriate choice for patients at higher risk who require chemotherapy. Sequential treatment with tamoxifen plus OFS followed by exemestane plus OFS might be a new area of research.

In summary, our comprehensive meta-analysis of all the published studies shows that addition of OFS does not appear to provide any additional benefits beyond those offered by adjuvant tamoxifen alone for premenopausal patients. Higher-risk patients who require chemotherapy might benefit from addition of OFS, but these results should be interpreted with caution because they were based on a smaller sample size and derived from only the SOFT and ZIPP trials. Further, exemestane plus OFS might be a better choice than tamoxifen plus OFS. Additional analyses and follow-up of the four studies might provide further insight into the use of OFS in combination with tamoxifen for the treatment of premenopausal women with early breast cancer.

\section{Acknowledgment}

This research was supported by a grant from the National Science Foundation of China (81302313).

\section{Disclosure}

The authors report no conflicts of interest in this work.

\section{References}

1. Siegel RL, Miller KD, Jemal A. Cancer Statistics, 2015. CA Cancer J Clin. 2015;65(1):5-29.

2. American Cancer Society. Breast Cancer Facts and Figures 2013-2014. Available from: http://www.cancer.org/acs/groups/content/@research/ documents/document/acspc-042725.pdf. Accessed February 11, 2015.

3. Pujol P, Daures JP, Thezenas S, Guilleux F, Rouanet P, Grenier J. Changing estrogen and progesterone receptor patterns in breast carcinoma during the menstrual cycle and menopause. Cancer. 1998;83(4): 698-705.

4. Parton M, Dowsett M, Smith I. Studies of apoptosis in breast cancer. BMJ. 2001;322(7301):1528-1532.

5. Osborne CK. Tamoxifen in the treatment of breast cancer. $N$ Engl J Med. 1998;339(22):1609-1618.

6. Klijn JG, Beex LV, Mauriac L, et al. Combined treatment with buserelin and tamoxifen in premenopausal metastatic breast cancer: a randomized study. J Natl Cancer Inst. 2000;92(11):903-911.

7. Mitsuyama S, Nomura Y, Ohno S, et al. [Assessment of goserelin treatment in adjuvant therapy for premenopausal patients with breast cancer in Japan - Zoladex Breast Cancer Study Group Trial-B]. Gan To Kagaku Ryoho. 2005;32(13):2071-2077. Japanese.

8. Baum M, Hackshaw A, Houghton J, et al; ZIPP International Collaborators Group. Adjuvant goserelin in pre-menopausal patients with early breast cancer: Results from the ZIPP study. Eur J Cancer. 2006; 42(7):895-904.

9. The Adjuvant Breast Cancer Trials Collaborative Group. Ovarian ablation or suppression in premenopausal early breast cancer: results from the international adjuvant breast cancer ovarian ablation or suppression randomized trial. J Natl Cancer Inst. 2007;99(7):516-525.

10. Cuzick J, Ambroisine L, Davidson N, et al. Use of luteinizing-hormonereleasing hormone agonists as adjuvant treatment in premenopausal patients with hormone-receptor-positive breast cancer: a meta-analysis of individual patient data from randomised adjuvant trials. Lancet. 2007; 369(9574):1711-1723.

11. Klijn JG, Blamey RW, Boccardo F, et al. Combined tamoxifen and luteinizing hormone-releasing hormone (LHRH) agonist versus LHRH agonist alone in premenopausal advanced breast cancer: a meta-analysis of four randomized trials. J Clin Oncol. 2001;19(2):343-353.

12. Crump M, Sawka CA, DeBoer G, et al. An individual patient-based meta-analysis of tamoxifen versus ovarian ablation as first line endocrine therapy for premenopausal women with metastatic breast cancer. Breast Cancer Res Treat. 1997;44(3):201-210.

13. Dowsett M, Cuzick J, Ingle J, et al. Meta-analysis of breast cancer outcomes in adjuvant trials of aromatase inhibitors versus tamoxifen. J Clin Oncol. 2010;28(3):509-518.

14. Tevaarwerk AJ, Wang M, Zhao F, et al. Phase III comparison of tamoxifen versus tamoxifen plus ovarian function suppression in premenopausal women with node-negative, hormone receptor-positive breast cancer (E-3193, INT-0142): a trial of the Eastern Cooperative Oncology Group. J Clin Oncol. 2014;32(35):3948-3958.

15. Francis PA, Regan MM, Fleming GF. Adjuvant ovarian suppression in premenopausal breast cancer. N Engl J Med. 2015;372(5):436-446.

16. Higgins JPT, Green S, editors. Cochrane Handbook for Systematic Reviews of Interventions Version 5.1.0. The Cochrane Collaboration, 2011. Available from: http://handbook.cochrane.org/. Accessed May 17, 2015. 
17. Higgins JP, Thompson SG. Quantifying heterogeneity in a metaanalysis. Stat Med. 2002;21(11):1539-1558.

18. Davidson NE, O'Neill AM, Vukov AM, et al. Chemoendocrine therapy for premenopausal women with axillary lymph node-positive, steroid hormone receptor-positive breast cancer: results from INT 0101 (E5188). J Clin Oncol. 2005;23(25):5973-5982.

19. Boccardo F, Rubagotti A, Amoroso D, et al. Cyclophosphamide, methotrexate, and fluorouracil versus tamoxifen plus ovarian suppression as adjuvant treatment of estrogen receptor-positive pre-/ perimenopausal breast cancer patients: results of the Italian Breast Cancer Adjuvant Study Group 02 randomized trial. J Clin Oncol. 2000; 18(14):2718-2727.

20. Paridaens RJ, Gelber S, Cole BF, et al. Adjuvant! Online estimation of chemotherapy effectiveness when added to ovarian function suppression plus tamoxifen for premenopausal women with estrogenreceptor-positive breast cancer. Breast Cancer Res Treat. 2010;123(1): 303-310.

21. Jakesz R, Hausmaninger H, Kubista E, et al. Randomized adjuvant trial of tamoxifen and goserelin versus cyclophosphamide, methotrexate, and fluorouracil: evidence for the superiority of treatment with endocrine blockade in premenopausal patients with hormone-responsive breast cancer - Austrian Breast and Colorectal Cancer Study Group Trial 5. J Clin Oncol. 2002;20(24):4621-4627.

22. Yang H, Zong X, Yu Y, et al. Combined effects of goserelin and tamoxifen on estradiol level, breast density, and endometrial thickness in premenopausal and perimenopausal women with early-stage hormone receptor-positive breast cancer: a randomised controlled clinical trial. Br J Cancer. 2013;109(3):582-588.

23. Cole MP, Jones CT, Todd ID. A new anti-oestrogenic agent in late breast cancer. An early clinical appraisal of ICI46474. Br J Cancer. 1971; 25(2):270-275.

24. Kisanga ER, Gjerde J, Schjøtt J, Mellgren G, Lien EA. Tamoxifen administration and metabolism in nude mice and nude rats. J Steroid Biochem Mol Biol. 2003;84(2-3):361-367.

25. Early Breast Cancer Trialists' Collaborative Group. Tamoxifen for early breast cancer: an overview of the randomised trials. Lancet. 1998; 351(9114):1451-1467.

26. Early Breast Cancer Trialists' Collaborative Group (EBCTCG); Davies C, Godwin J, Gray R, et al. Relevance of breast cancer hormone receptors and other factors to the efficacy of adjuvant tamoxifen: patientlevel meta-analysis of randomised trials. Lancet. 2011;378(9793): 771-784.

27. Davies C, Pan H, Godwin J, et al. Adjuvant Tamoxifen: Longer Against Shorter (ATLAS) Collaborative Group. Long-term effects of continuing adjuvant tamoxifen to 10 years versus stopping at 5 years after diagnosis of oestrogen receptor-positive breast cancer: ATLAS, a randomised trial. Lancet. 2013;381(9869):805-816.

28. Gray RG, Rea D, Handley K, et al. aTTom: Long-term effects of continuing adjuvant tamoxifen to 10 years versus stopping at 5 years in 6,953 women with early breast cancer. J Clin Oncol. 2013;31(18 Suppl): Abstr 5.
29. Schinzinger A. [About breast carcinoma]. Abstract presented at the 18th Congress of the German Society for Surgery. Zentralbl Chir. 1889;16 Suppl:55-66. German.

30. Early Breast Cancer Trialists' Collaborative Group (EBCTCG). Effects of chemotherapy and hormonal therapy for early breast cancer on recurrence and 15-year survival: an overview of the randomised trials. Lancet. 2005;365(9472):1687-1717.

31. Early Breast Cancer Trialists' Collaborative Group (EBCTCG). Systemic treatment of early breast cancer by hormonal, cytotoxic, or immune therapy. 133 randomised trials involving 31000 recurrences and 24000 deaths among 75000 women. Lancet. 1992;339(8784):1-15.

32. Arriagada R, Lê MG, Spielmann M. Randomized trial of adjuvant ovarian suppression in 926 premenopausal patients with early breast cancer treated with adjuvant chemotherapy. Ann Oncol. 2005;16(3): 389-396.

33. International Breast Cancer Study Group (IBCSG), CastiglioneGertsch M, O'Neill A, Price KN, et al. Adjuvant chemotherapy followed by goserelin versus either modality alone for premenopausal lymph nodenegative breast cancer: a randomized trial. $J$ Natl Cancer Inst. 2003; 95(24):1833-1846.

34. Recchia F, Saggio G, Amiconi G. Gonadotropin-releasing hormone analogues added to adjuvant chemotherapy protect ovarian function and improve clinical outcomes in young women with early breast carcinoma. Cancer. 2006;106(3):514-523.

35. Khan SS, Karn T, Symmans WF. Genomic predictor of residual risk of recurrence after adjuvant chemotherapy and endocrine therapy in high risk estrogen receptor-positive breast cancers. Breast Cancer Res Treat. 2015;149(3):789-797.

36. Arriagada R, Rutqvist LE, Mattsson A, Kramar A, Rotstein S. Adequate locoregional treatment for early breast cancer may prevent secondary dissemination. J Clin Oncol. 1995;13(12):2869-2878.

37. Fortin A, Larochelle M, Laverdière J, Lavertu S, Tremblay D. Local failure is responsible for the decrease in survival for patients with breast cancer treated with conservative surgery and postoperative radiotherapy J Clin Oncol. 1999;17(1):101-109.

38. Arimidex, Tamoxifen, Alone or in Combination (ATAC) Trialists' Group, Forbes JF, Cuzick J, Buzdar A, Howell A, Tobias JS, Baum M. Effect of anastrozole and tamoxifen as adjuvant treatment for early-stage breast cancer: 100-month analysis of the ATAC trial. Lancet Oncol. 2008;9(1):45-53.

39. Coates AS, Keshaviah A, Thurlimann B, et al. Five years of letrozole compared with tamoxifen as initial adjuvant therapy for postmenopausal women with endocrine-responsive early breast cancer: update of study BIG 1-98. J Clin Oncol. 2007;25(5):486-492.

40. van de Velde CJ, Rea D, Seynaeve C, et al. Adjuvant Tamoxifen and Exemestane in Early Breast Cancer (TEAM): a randomised phase 3 trial. Lancet. 2011;377(9762):321-331.

41. Pagani O, Regan MM, Walley BA, et al; TEXT and SOFT Investigators; International Breast Cancer Study Group. Adjuvant exemestane with ovarian suppression in premenopausal breast cancer. $N$ Engl J Med. 2014;371(2):107-118.
OncoTargets and Therapy

\section{Publish your work in this journal}

OncoTargets and Therapy is an international, peer-reviewed, open access journal focusing on the pathological basis of all cancers, potential targets for therapy and treatment protocols employed to improve the management of cancer patients. The journal also focuses on the impact of management programs and new therapeutic agents and protocols on

\section{Dovepress}

patient perspectives such as quality of life, adherence and satisfaction. The manuscript management system is completely online and includes a very quick and fair peer-review system, which is all easy to use. Visit http://www.dovepress.com/testimonials.php to read real quotes from published authors. 